\title{
Estimating Design Costs for First-of-a-kind Projects
}

\author{
Bakul Banerjee \\ Fermi National Accelerator Laboratory \\ POB 500 \\ Batavia, IL 60510
}

\begin{abstract}
Modern scientific facilities are often outcomes of projects that are first-of-a-kind, that is, minimal historical data are available for project costs and schedules. However, at Fermilab, there was an opportunity to execute two similar projects consecutively. In this paper, a comparative study of the design costs for these two projects is presented using earned value methodology. This study provides some insights into how to estimate the cost of a replicated project.
\end{abstract}

\section{INTRODUCTION}

A significant amount of basic and applied scientific research sponsored by U.S. government is conducted at several National Laboratories distributed throughout the country. Every year, the international scientific community benefits from major user facilities located at National Laboratories that are sponsored by Office of Science (SC) of U.S. Department of Energy (DOE). These laboratories are owned by DOE and operated by private organizations. These private organizations, also called contractors, have long histories of producing scientific breakthroughs by collaborating with universities around the world. With more than $\$ 400$ million dollars of annual budget, most of these laboratories have individuals with scientific and technical foresights to bring innovative concepts into fruition. National Laboratories continue to build and operate high-end special purpose facilities that could not be done by universities alone. Building or upgrading such facilities can cost billions of dollars. The cost of constructing first-of-a-kind facilities, using innovative materials, hardware, and software systems, is becoming increasingly expensive.

Recently, Fermilab had a unique opportunity to upgrade two similar systems consecutively for its RunII upgrade program. The first project was completed in 2005. The design phase of the second project is complete now. In this paper, a comparative study of the project cost for the design phases of both projects is presented. It is quite likely that a similar system will not be built again. However, an estimate of the scaling factors for the cost and effort for the first-of-a-kind and second-of-a-kind projects will be helpful for planning future projects. The lessons learned can be applied to other first-of-a-kind projects.

\section{MOTIVATION}

Since 1997, DOE sponsored various initiatives to improve project management processes within the department, as well as for its large network of contractors. The first step in this process was the execution of a baseline assessment done by a commission from the National Research Council. After the initial assessment was complete in 1999 [1], As a follow-up, DOE sponsored a series of three progress reports on implementation of recommendations from the assessment. The first progress report was published during the year of 2001. In the second report, released during 2002 [2], the assessment board paid particular attention to the first-of-a-kind and one-of-akind projects, often sponsored by SC. The board recognized that managing such projects with unique design concepts is difficult. However, public concerns for these increasingly expensive projects are also legitimate. As a custodian of public funds, DOE is obviously interested in assuring the public that these projects are executed in a financially responsible manner.

Since US government delegates the actual execution of large scale projects to contractors affiliated with public companies or universities, ultimate responsibilities for good project management practices lie with the private contractors. Contracting organizations often implement Earned Value Management System (EVMS) for all major projects, as well as to produce progress reports. According to recent government guidance, the compliance to EVMS standards is becoming critical even for medium size projects related to Information Technology (IT). Fermilab, a national accelerator laboratory, is owned by DOE SC. When necessary, the laboratory implements cost management methodologies that are described in the EIA standard EIA-748, "Earned Value Management Systems" [3].

A successful project depends on the front-end planning. A successful planning process requires decent history of similar projects. However, most projects sponsored by DOE SC are first-of-a-kind. By definition, there is no previous history for these projects. According to some experts, a first-of-a-kind project may take three times longer than a similar project in the industry. The primary objective for these projects is to implement major leap away from the existing technology. These projects may use cutting edge materials, many advanced electronics that may be in conceptual stage, or high-performance software that are yet to mature. In other words, these projects must implement "technologies of unproven field scale [1]". Since these projects may be in the planning stage for a long period, it is difficult to estimate the 
correct project cost since the pricing information for projected commercial technologies used may not be available. Also, the labor cost of designing innovative technologies, particularly for software intensive systems, is difficult to estimate. Since these projects are often very large with thousands of subprojects and tasks spread over many organizations, accurate history of required labor for even the more straight-forward components may not be readily available.

Fortunately, at Fermilab, we had an opportunity to execute two very similar projects of manageable scales consecutively. The same management and cost data collection methodologies are used for both projects. The engineering and scientific staff experience levels are also similar. Consequently, it is possible to perform accurate comparative analysis to glean insights into the general behavior of these projects.

\section{BACKGROUND}

Both projects considered for this study are associated with the development of new Beam Position Monitor (BPM) systems for two particle accelerators. The first project is for the Tevatron accelerator, a part of Fermilab's accelerator complex. This project, referred to as TevBPM, is a true first-of-a-kind project. Design risks involved with such a project are described in a previous paper [4] by the author. This project was initiated during August 2003 and ended in June 2005. The second-of-a-kind project, currently underway, is to implement a similar BPM system for the Main Injector accelerator. This project, referred to as MIBPM, started during July 2005 and almost all design activities are complete at the time of writing this paper. While the design work for the MIBPM project began within a month of the project initiation, it took about three months for system experts to establish requirements for the TevBPM system before the design work could begin. A life cycle phase based Work Breakdown Structure (WBS) was used for both projects. Details of the phase-based project organization are discussed in a previous paper [5]. The phase-based approach allows for isolating the cost of a particular phase from the rest of the project. Since none of the actual hardware cost is loaded to the WBS during the design phase, cost values extracted from the project plan reflect only the labor cost.

Both control systems serve beam position monitors located in underground tunnels dedicated to the specific accelerator. Above ground control systems are attached to these monitors using pickup cables. When accelerators are operational, signals received from the BPMs are processed and used to perform a number of control and diagnostic tasks. New systems are designed to capture and process both proton and antiproton signals from the accelerators. This significant enhancement was beyond the range of technical capabilities, both hardware and software, when the original accelerators were constructed. Engineers took advantage of exceptional progresses made in the hardware and software technologies. Scientists are delighted and compare the improvement TevBPM to a "new pair of glasses for the Tevatron."

As shown in the figure 1, both systems can be divided into two broad subsystems, specifically, electronic and software. The electronics subsystem includes a VME based hardware unit consisting of a timing module, multiple filter modules to capture and pre-process signals for further processing, and multiple special purpose Digital Receiver boards purchased from Echotek Corporation. The programmable timing module handling various clock events was developed in-house. This module, developed for the TevBPM, was enhanced for the MIBPM. For TevBPM, digital signals from the BPMs are pre-processed by filter cards residing in the VME crate. For MIBPM, each Echotek board is paired with analog pre-processing modules attached to analog raw signals that are housed in a stand-alone cage. Hardware components are integrated with the accelerator control software through the front-end software residing in corresponding VME processors. The front-end software is a VxWorks based data acquisition system. It performs preliminary acquisition and processing of data and communicates to the main accelerator control system.

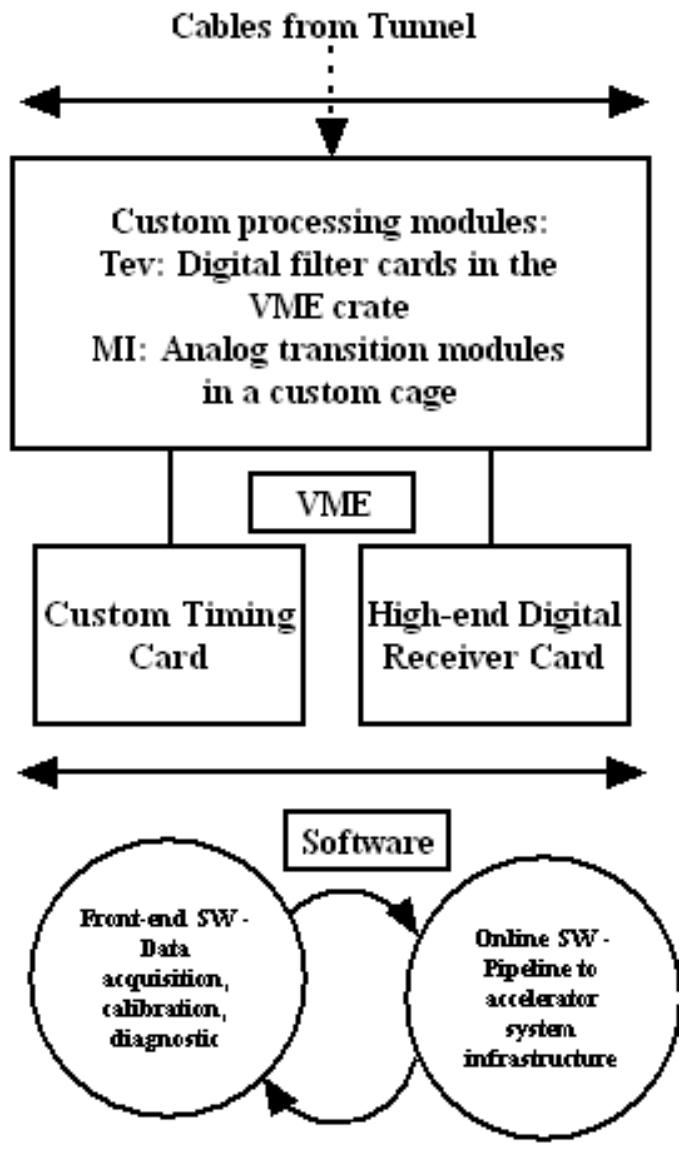

Fig. 1: BPM Subsystems

The software subsystem is composed of the front-end real-time software and the main accelerator control software. The front-end software, developed using modern methodologies, would remain easily extendable, 
maintainable, and reusable for future monitoring system projects. The online accelerator control software system provides accelerator operator interfaces, keeps track of the accelerator communication, system parameters and data. The main accelerator control software, also called online software, component of the project is mostly an enhanced version of the existing software. For both projects, modifications were made to software libraries and user interfaces.

Although both TevBPM and MIBPM systems are similar in many respects, the MIBPM system is significantly more complex. It has to handle interactions of the Main Injector with other accelerators. The TevBPM system had to deal with one signal category, while MIBPM has to deal with six different types of signals. The resulting increase in complexities of the front-end software and the timing module design is reflected in the cost of design. The design for the new analog signal pre-processing modules also required significant amount of work.

\section{COST Data ANALYSIS}

Although various project cost data was collected for both projects, we compare the EVMS measure called Estimate at Completion (EAC). The EAC measure for a particular task is defined to be the sum of the cost actually incurred up to the WBS status date and the cost estimated for remaining work to be done for that task. As described in [5], EAC seems to be an excellent measure for analyzing the performance of a project during a particular life cycle phase, provided that a life-cycle phase-based WBS model is used. EAC values are calculated on the end-of-themonth status date for the WBS. Since cost profiles for electronic and software subsystems are different, they are plotted separately.

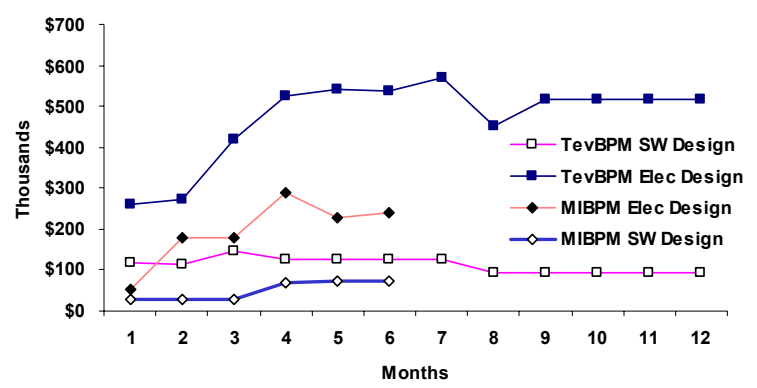

Fig. 2: Comparison for EAC in Design Phase

From figure 2, it is clear that it took close to eight months to complete the TevBPM electronic design. From the ninth month onward, the EAC graph flattens out. A flat EAC curve indicates that the work done during this period was in steady state and the amount of work is negligible if the task is nearing completion. In comparison, MIBPM electronic design took approximately five months of active work and about $50 \%$ of the cost of TevBPM work. Although there is a general expectation that the secondof-a-kind project should cost even lower, detailed design work may take longer than expected. Firmware enhancements often take longer than expected.

The EAC profile for TevBPM software design flattened out after eighth month, where as that of MIBPM flattens out after four months. On the other hand, the software design cost for MIBPM was almost $70 \%$ of the cost for TevBPM. This can be safely attributed to the complexities of the MIBPM system. However, the bulk of the design was complete within four month.

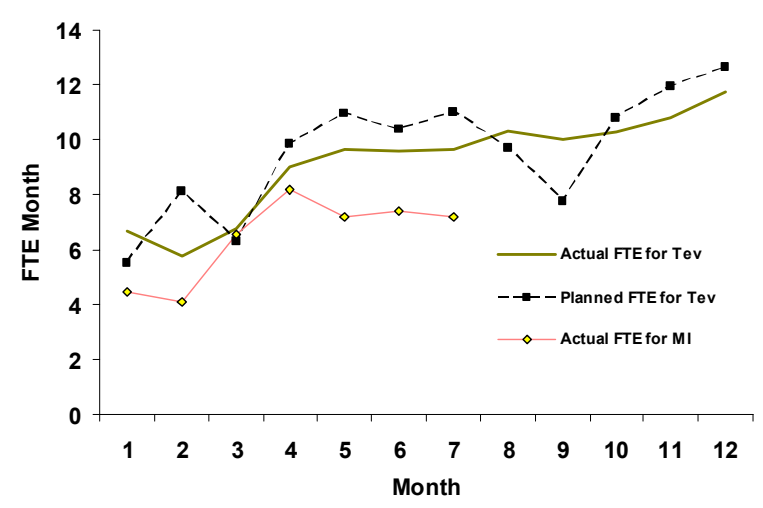

Fig. 3: Comparison of FTE Usage

In figure 3, the integrated efforts for both projects against the baseline plan for TevBPM are plotted. Integrated project effort for the first twelve months of the project includes efforts for definition of requirements, design, implementation and project management. The chart shows efforts in the units of one Full Time Employee (FTE) effort per month. As a first-of-a-kind project, the plan was somewhat pessimistic. However, the actual effort followed the general pattern of the plan. The total effort required for the MIBPM project is significantly smaller than that of TevBPM, which is to be expected.

\section{Conclusion}

As emphasized in the DOE progress report [2], the first step to the success of a first-of-a kind project is to gather and understand the technical specifications. However, successful project managers must also estimate the cost of the design and prototype efforts, since they may last for years. In a large project, many of the components may not be first-of-a-kind. They may be based on another first-ofa-kind project. This study shows a method of estimating the cost. Some of the conclusions that we can draw from this study are:

- For first-of-a-kind projects, beginning of the actual design phase may be delayed as the requirements definition may take longer than standard projects.

- For replicated projects, it is important to understand the magnitude of additional complexities. Significant increment of complexities can consume significant labor resources that may increase in an exponential 
manner. Weighting of complexities should be considered when assigning management reserves.

- Applying reusable and modular hardware, firmware, and software components can reduce the project cost significantly. Requirements for management reserves decrease with such components. The cost of introducing hardware, firmware, and software engineering tools can be paid for by the reduced cost of projects.

- Gathering historical EVMS data on reduced risk projects and analyzing them can be helpful. For a new project, analyzing similar data early in the project can provide valuable information on associated risks.

\section{ACKNOWLEGEMENT}

The author wishes to acknowledge her supervisor Dr. Stephen Wolbers and Fermi National Accelerator Laboratory, operated by Universities Research Association Inc. under Contract No. DE-AC02$76 \mathrm{CH} 03000$ with the United States Department of Energy.

\section{REFERENCES}

[1] Commission on engineering and Technical Systems, "Improving Project Management in the Department of Energy (1999)," National Academic Press

[2] Board on Infrastructure and the Constructed Environment, "Progress in Improving Project Management in the Department of Energy," National Academic Press

[3] EIA Standard EIA-748-A, "Earned Value Management Systems", January 2002

[4] B. Banerjee, "Managing Discovery Risks - A Tevatron Case Study," Proceedings of $5^{\text {th }}$ Annual UT/IEEE Engineering Management Conference, September, 2004

[5] B. Banerjee, "Applying EVM Principles to Tevatron Beam Position Monitor Project," Proceedings of IEEE Engineering Management Conference, September, 2005 